\title{
Reminder Care System: An Activity-aware Cross-device Recommendation System
}

\author{
May S. Altulyan ${ }^{1}$, Chaoran Huang ${ }^{1}$, Lina Yao $^{1}$, Xianzhi Wang ${ }^{2}$, Salil \\ Kanhere $^{1}$, and Yunajiang $\mathrm{Cao}^{1}$ \\ 1 UNSW Sydney, NSW 2052, Australia \\ m.altulyan@student.unsw.edu.au, \\ \{chaoran.huang, lina.yao, yuanjiang.cao\}@unsw.edu.au \\ , salilk@cse.unsw.edu.au \\ 2 University of Technology, Ultimo NSW 2007, Australia \\ $\{$ xianzhi.wang@uts.edu. au
}

\begin{abstract}
Alzheimer's disease (AD) affects large numbers of elderly people worldwide and represents a significant social and economic burden on society, particularly in relation to the need for long term care facilities. These costs can be reduced by enabling people with AD to live independently at home for a longer time. The use of recommendation systems in the Internet of Things (IoT) in the context of smart homes can contribute to this goal. The Reminder Care System (RCS) described in this paper is an activity-aware cross-device recommendation system that aims to forecast the devices/objects Alzheimer's patients will use by analysing their interactions with sensory objects and environments. In this paper, we present RCS, a research prototype of a recommendation system in IoT for elderly people with cognitive disabilities. RCS exploits daily activities that are captured and learned from IoT devices to provide personalised recommendations. The experimental results indicate that RRCS can inform the development of real-world IoT applications.
\end{abstract}

Keywords: Smart home - Internet of Things. recommender systems. activity recognition.

\section{Introduction}

Alzheimer's disease (AD) is an incurable degenerative disease that affects the brain cells and has serious implications for large numbers of elderly people worldwide. In the United States, for instance, 6.08 million older people had mild cognitive impairment or clinical AD in 2017 and this figure is projected to increase to 15.0 million by 2060 [7]. AD can be broadly categorized into three stages: mild (which can last around 3 years); moderate (also around 3 years); and the final severe stage. During the mild stage, patients begin to lose short-term memory, which affects their ability to remember people's names or recent events. In the moderate stage, patients may experience acute memory loss, which renders them unable to handle simple tasks without help from others, as well as language 
problems, confusion about time, personality change and emotional lability. In the severe stage, patients lose the ability to talk, understand, swallow and walk and require intensive care from professional or family caregivers $[1,11]$.

Despite advances in medical technology, AD remains incurable and its aetiology is not fully understood. As a result, long-term care for Alzheimer's patients is a major financial burden for society. In 2018, the total cost of caring for Americans with Alzheimer's disease and other dementia was estimated at $\$ 277$ billion [4].

One approach to addressing this issue is to deploy recent advances in sensor network technologies, in particular the emerging Internet of Things (IoT). These technologies have the computing ability to support a wide range of smart home applications that may enable people with $\mathrm{AD}$ to live independently for a longer time, thus reducing the costs associated with institutional care, particularly in the early stages. Most currently available smart home applications focus on monitoring the elderly with cognitive disabilities by providing intelligent ambient environments that can detect accidents or symptoms $[3,6,16,20,26,28]$. However, less attention has been paid to developing smart applications that support Alzheimer's patients by recommending the sequences of tasks needed to complete 'activities of daily living. Such applications can also improve their social health by enabling them to manage their life with some degree of independence.

Recommendations systems in the IoT environment can play a crucial role in helping Alzheimer's patients to remain living independently in their own homes. The following scenario presents an example of how such a system could operate by tracking the person's routine daily activities.

Motivating Scenario. Alice is a 79-year- elderly women with Alzheimer's disease who lives alone in a house. She is making a cup of coffee and her kitchen knows it. Motion sensors monitor her every move and track each coffee-making step. If she pauses for too long, a reminder application reminds her about what to do next. If she tries to prepare a cup of coffee late at night, the system will remind her of the time and recommend her to go back to bed. Later that day, Alice's son can access the secure application and scan a checklist that has been created from the phone in his mother's house. He finds that his mother took her medicine on schedule, slept and ate normally, and continued to manage her daily activities on her own.

A basic question emerging from this scenario is whether there currently exists a recommender system in smart environments that can exploit different data sources to provide reminder recommendations. If so, what advances in activity recognition and learning algorithms do we need to harness the combined power of these diverse sources? In order to provide reminder recommendations for these complex activities, we need to exploit three major sources of information: simple human activities, localization, and object usage detection. This is the motivation for our research. While the idea of designing a recommender system specifically for Alzheimer's patients is certainly not new, our approach is unique because we explicitly consider multi-source data instead of relying exclusively on people's direct interaction with the technologies [18]. The existing recommendation models 
cannot be directly applied to our scenario, because they either overlooked at the context, or they only include the contextual signals in a fixed combination from physical environments with ignorance of contemporary user's activity $[9,22,25]$.

In this case, the key challenge is to effectively disambiguate the associations among these sources and to identify an algorithm that exploits this relationship to produce reminder recommendations. We design an unobtrusive Reminder Care System (RCS) that helps patients, especially those in the early stages of $\mathrm{AD}$, to achieve their goals by guiding them through their activities. RCS consists of two main stages. First, we infer people's activities by analyzing three main data sources (simple human activities, localization and object usage detection). Second, we adopt probabilistic matrix factorization which exploits these data to provide remind recommendations.

In this paper, we present the design and development of a prototype system, RCS, for a smart home monitoring process that produces remind recommendations. RSC has the ability to automatically learn the contexts in a smart home environment, such as object usage and daily human activity, by analyzing the motion sensor data generated by human movements and interactions with objects. It enhances Alzheimer's patients' awareness of their surroundings and provides better recommendations. In addition, the proposed system enables users to create personal rules through a graphical interface by exploiting the interaction with IoT-enabled devices, objects, and contextual events. Several IoT applications can benefit from the architecture and implementation of our system. Its main contributions are discussed below.

\section{Related Work}

In this section, we provide an overview of (1) smart home applications that aim to provide assistance for older adults and (2) recommender systems in IoT that are closely related to our approach.

Smart home applications a great deal of research has sought to identify and exploit Activities of Daily Living (ADLs) in the smart environment to help elderly people to complete their activities or reach their goal. [25] present an endto-end web-based in-home monitoring system, namely WITS, for convenient and efficient care delivery. The system exploits both the data- and knowledge-driven techniques to enable a real-time multi-level activity monitoring in a personalized smart home. [2] exploited a fuzzy temporal data-driven technique to design an anomalies recognition and assistance provision system. The system can characterise the activity into normal or anomalous. Each activity is defined as a fuzzy conceptual structure which is represented as a hierarchy of concepts in a smart home. The base of the hierarchy represents the observations, while normal world generic function at the top of the hierarchy represents the normal world. To detect if the observed activity is anomalous or not, the distance between the observed activity and the normal world is calculated. Accordingly, the system works to restore the normal state of the smart home. [11] proposed a SmartMind system to monitor the activity of Alzheimer's patients. It focuses on dividing the 
smart area into two regions, safe and unsafe, and expects patients to perform the activities only in the safe region. If the system detects that the patient is outside the safe region, an alert is activated, and a notification is sent to the patient's relative to take the necessary action. However, this system has several limitations in addition to cost. The patient still depends on the assistance of a caregiver to guide them to complete their activities or reach their goal. Further, the system does not consider that some activities performed inside the boundary may be unsafe. For example, due to memory loss, the patient might forget to turn off the stove after preparing a meal; in this situation, the relative would not receive a notification. [18]proposed a system, SML, to track the indoor daily activities of elderly people with mild cognitive impairment. It aims to recommend the correct sequence of tasks for each activity to ensure that the user can reach his goal. It is based on the situ-framework which represents each activity as a sequence of situations and a situation consists of three tuples $\langle M, B, E\rangle$ where $M$ is the patient's mental state, $B$ represents the behaviour context ,and $E$ is the environmental context.The recommendation is based on applying a reinforcement learning algorithm that enables a decision to be made even with incomplete knowledge. Latfi et al. proposed an ontological architecture which consists of seven ontologies for the Telehealth Smart Home. It aims to build high-level intelligent applications, particularly for older people suffering from loss of cognitive autonomy [12]. In our work, in addition to exploiting the interaction between user and things, we extend the model to the relational network of three main data sources: localization, user and thing interaction and simple activities. This improves performance in detecting latent features from the relational network of these sources that can be used to define complex activities.

Recommender system (RS) in IoT provide a critical platform for demonstrating the benefits of the Internet of Things (IoT). However,it faces several challenges that are more complex than those associated with the conventional recommender approach, for three main reasons: (1) it deals with and analyses a massive amount of extremely heterogeneous data; (2) it needs to exploit rich contextual information; and (3) the data have to pass through several layers for extensive processing over the entire life cycle. Accordingly, numerous studies have proposed various methods for recommender systems in IoT. Hwang et al. proposed a method which aims for generating automatic rules and recommending the best rule based on things that are installed in smart homes.Accordingly, the user has the opportunity to add new rules based on any new devices that are connected to the home without extra efforts. To investigate this goal, the authors applied two steps, designed three ontology models and applied data pipeline based on open data [9]. Salman et al. exploited context aware to build a proactive multi-type context-aware recommender system which provides different kinds of recommendations. This system has two main phases: identifying the situation of the user and the type of recommendations, and recommending the item [22]. Sewak and Singh designed the Optimal State based Recommender (OSR) System by exploiting some machine learning algorithms such as Distributed Kalman Filters; Distributed Mini-Batch SGD (Stochastic Gradient Descent) ; and Dis- 
tributed Alternating Least Square (ALS) method. It shifted the conventional recommendations that are based on user/ item preferences only into accurate recommendations dealing with real-time. [23]. The most important feature of such a recommender system in IoT is its ability to exploit knowledge of human behaviour and the relationships between things as sources to produce accurate recommendations [27]. Massimo proposed inverse reinforcement learning (IRL) to model user behaviour to improve the quality of recommendations. The use of IRL in recommender systems has two main features: it learns user decision behaviour by observing the user's actions; and it can learn even from a few samples by grouping its observations [15]. As well, providing personal recommendations makes the user more interested in continuing, especially since most of the recommender systems are smart phone applications. Concerns about limited battery capacity might lead users to turn off this application if its recommendations do not match their preferences. $[5,10]$ built recommender systems to conduct personalised recommendations by exploiting several data sources. Yao et. al [27] propose a unified probabilistic factor based framework by fusing social, temporal and spatial relations across heterogeneous entities of IoT to make more accurate recommendations. Our proposed approach has two obvious advantages over the related work discussed above:(1)the contexts in a smart home environment can be learnt automatically by the system (2) users have ability to create personal rules through a graphical interface by exploiting the interaction with IoT-enabled devices, objects,and contextual events.

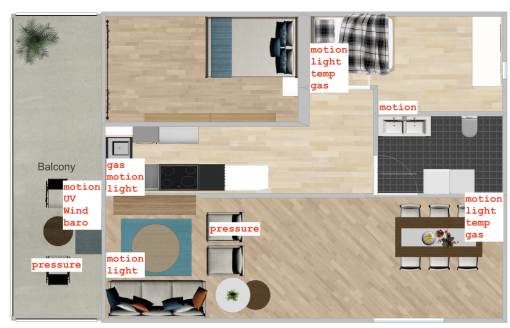

Fig. 1: Example of sensor deployment in home environment

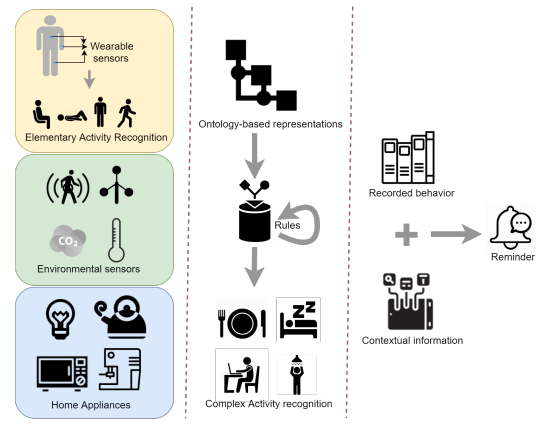

Fig. 2: Overview of the proposed methodology

\section{Reminder Recommender Care System}

In this section we describe the three major part(see Fig. 2) of our proposed system for reminder recommendation: (1) Correlation analysis of devices, where we construct a general ontological model for representing human domestic activities, domain concepts and objects; (2) Rule-based orchestration, where strategy 
will be developed based on a semantic distance-based rule matching method and rule generation; and (3) Activity-triggered Recommendation, which utilized matrix factorization technique to exploit information learnt in the previous two steps, to give reminder recommendations to users.

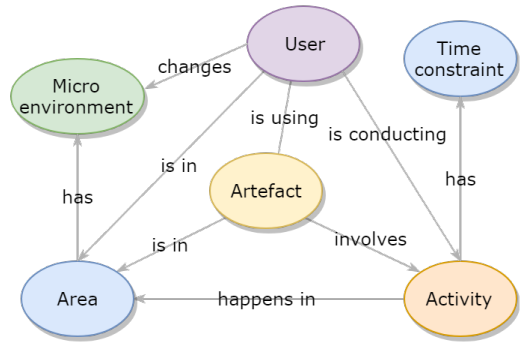

Fig. 3: Activity area modeling

\begin{tabular}{ll}
\hline Elementary activities & Complex activities \\
\hline Lying & Sleeping \\
Sitting & Watching TV \\
Standing & Preparing tea \\
Walking & Cooking \\
Running & Calling phone \\
& Eating \\
& Taking medicine \\
\hline
\end{tabular}

Table 1: Example of activities

\subsection{Correlation analysis of devices}

Intuitively, as a system to recommend reminders to user, we need to firstly understand what activity or activities our user is undertaking in relatively high level, that is, a Human Activity recognition(HAR) task.

Studies on HAR has been studied extensively, yet the most ones are still simple or elementary activity recognition, especially with wearable sensors. And elementary activity recognition often only reveal limited information about the user, which can hardly meet the requirements of complex practical applications. Thus, there emerging studies on complex activities recently. And some [14,25] has shown that decomposing complex activities into basic or elementary activities works well. Here we also adapt this strategy, to exploit the recognized elementary activities as one source of our input, along with environmental sensory(see Fig. 1) data and the usages of home appliances, to reveal the correlations of devices.

Definitions Our input sensory data is continuously streaming, with a certain sample rate limited by hardware. We denote the data reading $d$ from sensor $\sigma$ at timestamp $t$ as a tuple $r=\langle t, \sigma, d>$. Accordingly we denote a recent set of reading of sensor $\sigma$ in a duration between timestamp $t_{1}$ and $t_{2}$ as $R_{t_{1}, t_{2}}^{\sigma}=$ $\left\{r_{1}, r_{2}, \ldots, r_{n}\right\}, n \in \mathbb{R}$, is the number of timestamps.

Given a recent set of sensory data readings $R_{t_{1}, t_{2}}^{\mathcal{S}}$ where $\mathcal{S}=\left\{\sigma_{1}, \sigma_{2}, \ldots, \sigma_{m}\right\}$ denotes a set of sensors in this reading, our task is now firstly recognize complex activities set $\mathcal{A}=<A_{1}, A_{2}, \ldots, A_{k}>$ happened within the time range $\left(t_{1}, t_{2}\right)$, and then make recommendations of reminders $\Gamma=<\gamma_{1}, \gamma_{2}, \ldots, \gamma_{l}>$. 
Elementary activity recognition Here we assume to follow the set up of PAMAP2 [19], using 3 wearable inertial measurement units(IMUs) as data source. The IMUs in the setting can collect 3D-acceleration data, 3D-gyroscope data, and 3D-magnetometer data. Also as illustrated in left upper part of Fig. 2, the IMUs are deployed on the chest, wrist, and ankle of our subject. Here we considering first 5 status related elementary activities in PARMAP setup, namely: lying, sitting, standing, walking, running(see Table ??). Given a series of readings $R_{t_{1}, t_{2}}^{\mathcal{S}_{\mathcal{M} U}}=\left\{r_{1}, r_{2}, \ldots, r_{j}, \ldots, r_{n}\right\}, r_{j} \in \mathbb{R}^{K}$, where $K$ is the number of IMU sensory data we collect at one timestamp, in this step we require an series output of elementary activities $\alpha=\left\{a_{1}, a_{2}, \ldots, a_{b} s\right\}$ accordingly. As here the input is time series signals from multiple IMUs, DeepConvLSTM [17] classifiers have been proved powerful in handling such data. Note that for such a classifier, the input is a batch of $n$, and here we set it to be the number of data we collect at one certain time duration with an overlap.

DeepConvLSTM is a state-of-the-art of deep neual networks which combines convolutional, recurrent and softmax layers. The ReLU Convolutioanl layers firstly take the size- $n$ squencial batch inputs over time $\left[t_{1}, t_{2}\right)$, and they produce a 2D feature map of size $\eta \times K \times f$. Here $f$ denotes the number of filters. The feature map is then received by 2 dense recurrent layers, which in here is made up with LSTM cells, and finally a softmax layer outputs the prediction results.

More details of our settings for this classifier can be found in the Experiment section later.

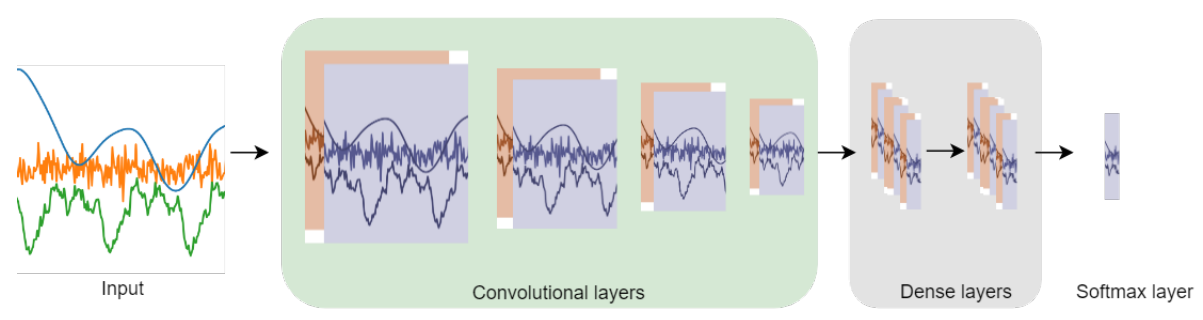

Fig. 4: The structure of DeepConvLSTM

Ontology for complex activity recognition To our knowledge, it is Yamada et al. [24] firstly come out the idea to apply ontology model in HAR, that involves interactions, i.e., subject performs activities that involves interactions with certain things. And such interactions often result in changing in status of things and environment, produce some contextual information. For example, boiling water can result in 1) local environmental changes like motion(IR radiation by human) and locally high humidity in the kitchen, 2) triggering the usage status of the kettle, 3) time constraint as such activity is highly unlike to happen 
in early morning before sunrise. In this example we can observe firstly spatial contexts: motion detected in kitchen, that is the place this activity happens; secondly artifactual context: the kettle has been used; thirdly the environmental contexts: the risen in humidity.

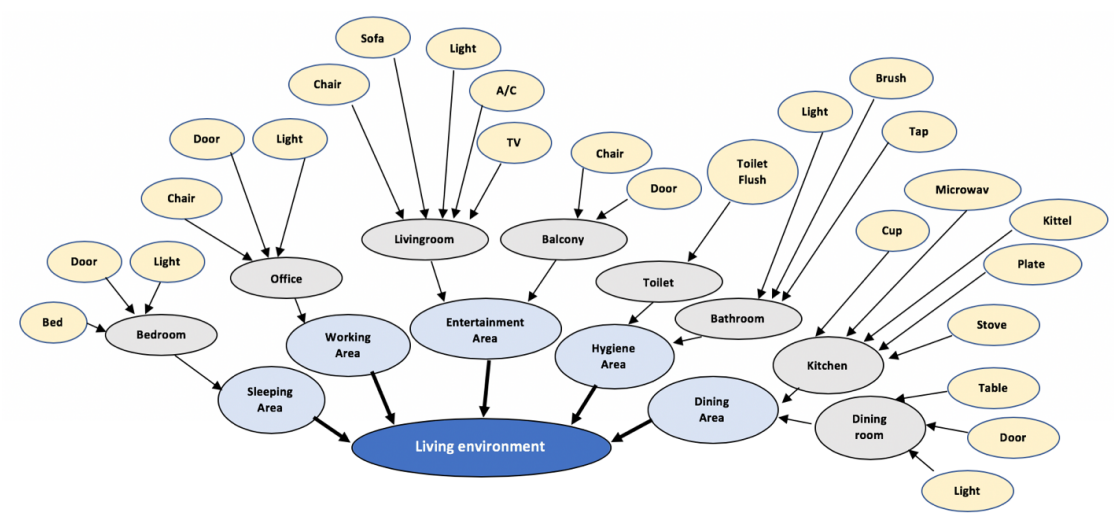

Fig. 5: Example of living area ontology

Noted that in our settings there are some more context information that can help further: in subsection 3.1, we utilized wearable IMUs and LSTM recognized elementary activities of our subject: and in this example, it outputs some activities include while not limited to standing and arm motions. This basic model of activity with area, environment and time constraint can be presented as Fig. 3, and Fig. 5 shows the relationships between artefacts and area.

Accordingly, we build a OWL(Ontology Web Language ${ }^{3}$ ) ontological models to help recognize complex activities, which include ontological models for artefacts, environment, locations, and activities. And Fig. 5 shows the ontology for location, which represents relationships between artefacts and area.

Noted here we have relatively manageable number of sensors deployed, which makes us possible to manually create instances. As for the collected data, we largely have sensors with binary outputs: PIR sensor indicates the absence or presence of human, i.e. user, in certain area with a 0 or 1 readings; electronic valves status indicates if water tap is running. Even sensors with complex value, we can still using simple rules to map the reading into standard semantic presentation. For example, UV meter with reading less than 3 stands for low, between 3 and 5 can be interpret moderate, etc.; Temperature/barometer sensors indicates temperature/pressure rise or fall.

\footnotetext{
$\overline{3}$ https://www.w3.org/standards/techs/owl
} 


\section{$3.2 \quad$ Rule-based orchestration}

This part is mainly use previous collected sensory data and ontological models, to recognize the complex activities the user is performing. It contains set of rules, which is extended from the ontological models and combined constraints. The rules response accordingly with set of inputs, and output recognized activities, following the knowledge carried by ontological models and semantically represented sensory data, along with elementary activities within the sensed duration.

Given our ontological models built, rules to recognize complex activities can be produced accordingly, which includes artefacts involved in this activity, area that the activity taken in place, key elementary activities that this activity contains, local environmental changes and the time constraint.

Following the example of activity boiling water above, we can set the ontological rule in descriptive language as:

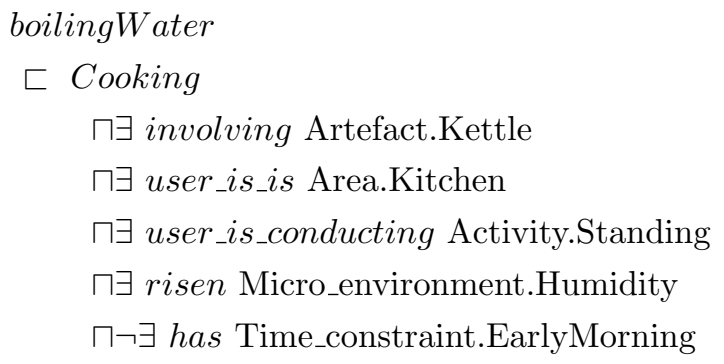

Similarly, by traversing the ontological models, we can build rules for all defined activities.

\subsection{Activity-triggered Reminder Recommendation}

Early works for activity-triggered reminder recommendation usually using Fuzzy Logic to make decision. While studies show these approaches can work adequately, they can still be improved. One shortcoming is the logic is basically predefined and they may either unable to handle undefined scenarios or the system can be too complex to deal with unpredicted scenarios.

Collaborative Filtering technique, especially Matrix Factorization, has been widely applied in recommender systems, while limited works in reminder recommendation can be identified. Here we propose to use Non-negative Matrix Factorization(NMF) [13] as our predictor for reminder recommendation.

Activity $A$ is already been recognized in previous steps, in addition to the descriptive ontology $O$ for activity $A$ with properties set $P$, and now for reminder $\gamma$, user have a rating $\rho$. After certain period of time, we can easily collect a set of activities records $\mathcal{A}$, related ontologies $\mathcal{O}$ with properties $\mathcal{P}$ and accordingly for a set of reminder $\Gamma$ with ratings $\varrho$. For simplification, we note the matrix $[A ; \Gamma ; P ; \varrho]$ as $M$, the NMF has goal to decompose $M$ so that $M \approx V H$, which 
can be achieved by minimizing the error function:

$$
\min _{V H}|| M-V H \|_{F}, \text { subject to } V, H \geq 0
$$

For $M^{\prime}=V H$, we restore activities $\mathcal{A}$, reminders $\Gamma$ and learned ratings $\varrho^{\prime}$, where the rank $\rho$ 's to $[A ; \Gamma]$ is the list of our recommendations of reminders. Accoring to the rounded $\rho$ 's, we issue the users with high priority reminders.

However, the system still struggles with cold-start. Common solution will be let the user give ratings for random reminders for a certain period of time, which is arguably undesirable. Here we exploit a previous study by Zhou et al. [29], where a survey is done and they have some ground truth on certain ratings under 20 different scenarios. This provision can help to boost our system in cold-start. We derived the data presented in their paper ${ }^{4}$, and Table 2 shows the data.

\begin{tabular}{rllr}
\hline ID & Current activity & Triggered reminder & Rating \\
\hline 1 & Sleeping & Taking medicine & 4 \\
2 & Sleeping & Washing clothes & 1 \\
3 & Sleeping & Turning off gas & 5 \\
4 & Sleeping & Cooking & 2 \\
5 & Answering phone & Taking medicine & 2 \\
6 & Answering phone & Washing clothes & 2 \\
7 & Answering phone & Turning off gas & 5 \\
8 & Answering phone & Cooking & 2 \\
9 & Watching TV & Taking medicine & 4 \\
10 & Watching TV & Washing clothes & 2 \\
11 & Watching TV & Turning off gas & 5 \\
12 & Watching TV & Cooking & 4 \\
13 & Wandering & Taking medicine & 4 \\
14 & Wandering & Washing clothes & 4 \\
15 & Wandering & Turning off gas & 5 \\
16 & Wandering & Cooking & 4 \\
\hline
\end{tabular}

Table 2: Default reminders with rating (derived from Zhou et al. $[29]^{4}$ )

\section{Evaluation}

In this section we present systematic evaluation of our proposed reminder recommender system, via experiments on public datasets. As the proposed system consists of 3 major parts(see Fig. 2), the evaluation is conducted accordingly.

We will first introduce datasets used for evaluation, and then presents experiment settings and evaluation and result analysis.

\footnotetext{
4 Here we use "user expectation level of reminder (UEL)" as rating and 5 levels converted to numbers(5 stand for "very high" and respectively).
} 


\subsection{Dataset}

We evaluate the proposed system on well-benchmarked public dataset. Firstly we evaluated our elementary activity recognition module, since the recognized elementary activities are used as one of the inputs of our second process stage, it is essential to ensure its performance. This is evaluated with both PAMAP2 dataset [19] by UC Irvine and also the PUCK dataset $[8,21]$ in Washington State University(WSU) CASAS project in 2011.

After that, the system proceed to complex activity recognition, and this is mainly evaluated on the PUCK dataset, as it contains both smart home environmental sensors and wearable sensory data from smartphones, which match exactly our needs.

PAMAP2 dataset The PAMAP2 dataset contains 9 participants performing 12 daily living activities including both basic actions and sportive exercises. The activity sensory data is collected from 3 Inertial Measurement Units (IMUs) attached to three different positions, namely the dominant wrist, the chest and the dominant sides ankle. Each IMU contains two 3-axis accelerometers, two 3 -axis accelerometers, one 3 -axis gyroscopes, one 3 -axis magnetometers and one thermometer with sampling rate of $100 \mathrm{~Hz}$.

PUCK dataset The PUCK dataset ${ }^{5}$ is collected in Kyoto smart home testbed in Washington State University, and it is a two-story apartment with one living room, one dining area, one kitchen, one bathroom and three bedrooms. Various environmental sensors are installed in this testbed, including motion sensors on ceilings, door sensors on room entrances, kitchen cabinet doors as well as microwave and refrigerator doors, temperature sensors in rooms, power meter, burner sensor, water usage sensors, telephone usage sensors and some item sensors for usage monitoring.

\begin{tabular}{rcc}
\hline Method & PAMAP2 & PUCK \\
& & \\
\hline SVM & 58.7 & 63.4 \\
KNN & 69.3 & 71.8 \\
Random Forest & 62.8 & 72.1 \\
LSTM & 71.9 & $\mathbf{7 7 . 4}$ \\
DeepConvLSTM & $\mathbf{7 3 . 4}$ & 77.2 \\
\hline
\end{tabular}

Table 3: Baseline test of elementary activity recognition

It is worth mentioning that there is also mobile wearable sensor data available for some subjects, in similar settings of PAMAP2, although less in the numbers

\footnotetext{
${ }^{5}$ http://casas.wsu.edu/datasets/puck.zip
} 
of sensors. And in this work, the annotated ground truth is rather rare, we combined subject 3,8 and 10 , because they are the only source of data where environmental and wearable sensor both available publically. We identified 5 complex activities in this dataset, namely 16 instances of Making phone call, 4 instances of Washing hands, 4 instances of Cooking, 4 instances of Eating and 4 instances of Cleaning kitchen. Noted that with our ontology based rules, some more activities such as Sleeping, Wandering can be easily identified as well, yet not quite suitable for evaluation.

\subsection{Elementary activity recognition}

As mentioned, firstly we studied the effectiveness of DeepConvLSTM on our tasks, to identify the geusture of subject, that is the elementary activities. We tested sevral baselines, including $\mathrm{SVM}, \mathrm{kNN}(\mathrm{k}=3)$, Random Forest(128 trees) and ordinary LSTM with 3 layers and 64 cells. As for the DeepConvLSTM, we adpated a common configuration, where there are 4 convolutional layers with feature maps, 2 LSTM layer with 128 cells. All above tests are conducted with the same settings on both PAMAP2 and PUCK datasets. The above results(in Table 3) prove that our choice of DeepConvLSTM, which outperform all tested baselines on PAMAP2 and adequate on PUCK, where the gap is so small that can be arguably ignored.

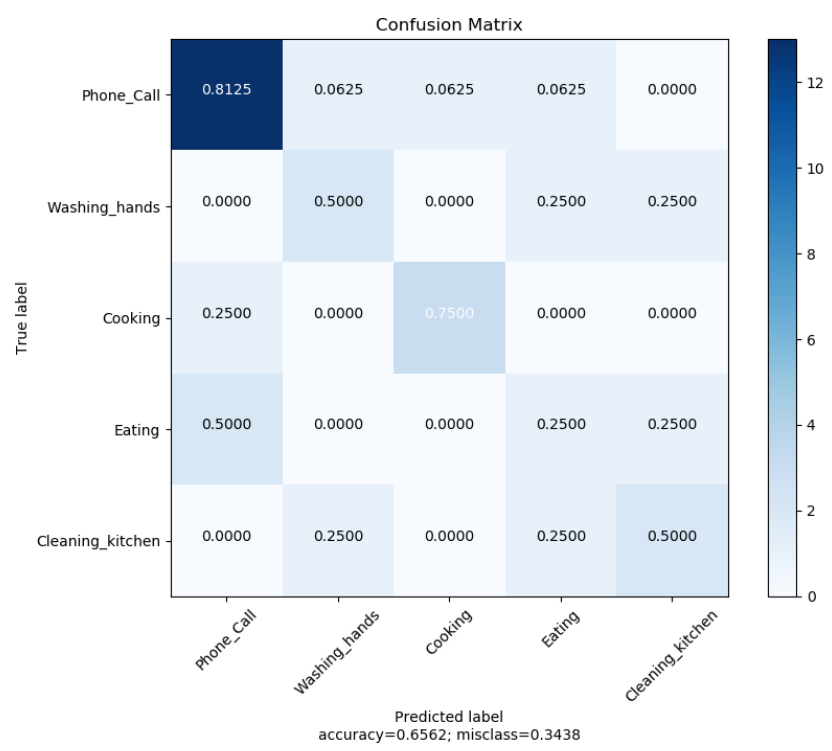

Fig. 6: The confusion matrix of complex activity recognition 


\subsection{Complexity Activity recognition}

Given our ontology based rules ready, we can perform complex activities recognition. The tests result is reported in Fig 6.

Here we can easily read that the overall accuracy for complexity activity recognition is of $65.625 \%$, which is quite reasonable, especially considering that this is based on elementary activity recognition, where our accuracy is around $77.2 \%$, as well as the sample is still quite rare. Also we can tell our framework tend to mis-classify Phone call and Eating, Washing hands and Cleaning kitchen. This may be caused by the sharing characteristics of those activities where similar places and devices are being used.

\section{Conclusion}

In this work, we explored the feasibility of combining environmental sensors and wearable sensors, to recommend reminders to the people need extra care, especially Alzheimier's patient, in an activity-aware and cross-devices basis. We take advantage of one state-of-the-art HAR recognition approaches, DeepConvLSTM to identify the gestures of subject, combining which with predefined ontology representations the environmental senorary data, to produce rules for complexity activity recognition. And this output of subject behaviours are feed into a proposed recommendation engine, and output reminders to the user accordingly. The proposed recommeder system also will retrain itself given appropriate feedback from the user. Experiments demonstrated that our behaviour recognition part is effective and hence the recommendation engine practicable.

However, our evaluations of this system is still not comprehensive enough to prove the premium of this framework, mainly due to lack of test data sources to fit our complex experiments need. In the feature, we will build a running system with a sensor enabled test-bed, to collect inclusive and adequate data, and test our framework in reallife use case.

\section{References}

1. Alzheimers society, https://www.alzheimers.org.uk/about-dementia/ types-dementia/alzheimers-disease-symptoms\\#content-start, accessed 2019-01-07

2. Amirjavid, F., Bouzouane, A., Bouchard, B.: Intelligent temporal data driven world actuation in ambient environments: Case study: Anomaly recognition and assistance provision in smart home. In: Computer and Information Science (ICIS), 2013 IEEE/ACIS 12th International Conference on. pp. 287-293. IEEE (2013)

3. Aramendi, A.A., Weakley, A., Schmitter-Edgecombe, M., Cook, D.J., Goenaga, A.A., Basarab, A., Carrasco, M.B.: Smart home-based prediction of multi-domain symptoms related to alzheimer's disease. IEEE Journal of Biomedical and Health Informatics (2018)

4. Association, A., et al.: 2018 alzheimer's disease facts and figures. Alzheimer's \& Dementia 14(3), 367-429 (2018) 
5. Asthana, S., Megahed, A., Strong, R.: A recommendation system for proactive health monitoring using iot and wearable technologies. In: AI \& Mobile Services (AIMS), 2017 IEEE International Conference on. pp. 14-21. IEEE (2017)

6. Blount, M., Ebling, M.R., Eklund, J.M., James, A.G., McGregor, C., Percival, N., Smith, K., Sow, D.: Real-time analysis for intensive care: development and deployment of the artemis analytic system. IEEE Engineering in Medicine and Biology Magazine 29(2), 110-118 (2010)

7. Brookmeyer, R., Abdalla, N., Kawas, C.H., Corrada, M.M.: Forecasting the prevalence of preclinical and clinical alzheimer's disease in the united states. Alzheimer's \& Dementia 14(2), 121-129 (2018)

8. Das, B., Cook, D.J., Schmitter-Edgecombe, M., Seelye, A.M.: Puck: an automated prompting system for smart environments: toward achieving automated prompting-challenges involved. Personal and ubiquitous computing 16(7), 859$873(2012)$

9. Hwang, I., Kim, M., Ahn, H.J.: Data pipeline for generation and recommendation of the iot rules based on open text data. In: Advanced Information Networking and Applications Workshops (WAINA), 2016 30th International Conference on. pp. 238-242. IEEE (2016)

10. Kamal, R., Lee, J.H., Hwang, C.K., Moon, S.I., Hong, C.S.: Autonomic inferring of $\mathrm{m} 2 \mathrm{~m}$-iot service-usage from user-emotion and environmental information. Proceedings of the Korea Information Science Society pp. 1034-1036 (2013)

11. Lam, K.Y., Tsang, N.W.H., Han, S., Ng, J.K.Y., Tam, S.W., Nath, A.: Smartmind: Activity tracking and monitoring for patients with alzheimer's disease. In: Advanced Information Networking and Applications (AINA), 2015 IEEE 29th International Conference on. pp. 453-460. IEEE (2015)

12. Latfi, F., Lefebvre, B., Descheneaux, C.: Ontology-based management of the telehealth smart home, dedicated to elderly in loss of cognitive autonomy. In: OWLED. vol. 258 (2007)

13. Lee, D.D., Seung, H.S.: Learning the parts of objects by non-negative matrix factorization. Nature 401(6755), 788 (1999)

14. Liu, L., Cheng, L., Liu, Y., Jia, Y., Rosenblum, D.S.: Recognizing complex activities by a probabilistic interval-based model. In: AAAI. vol. 30, pp. 1266-1272 (2016)

15. Massimo, D.: User preference modeling and exploitation in iot scenarios. In: 23rd International Conference on Intelligent User Interfaces. pp. 675-676. ACM (2018)

16. Nelson, T.W.H., Kam-Yiu, L., Joseph, N.K.Y., Song, H., Ioannis, P.: Tracking indoor activities of patients with mild cognitive impairment using motion sensors. In: Advanced Information Networking and Applications (AINA), 2017 IEEE 31st International Conference on. pp. 431-438. IEEE (2017)

17. Ordóñez, F., Roggen, D.: Deep convolutional and lstm recurrent neural networks for multimodal wearable activity recognition. Sensors 16(1), 115 (2016)

18. Oyeleke, R.O., Yu, C.Y., Chang, C.K.: Situ-centric reinforcement learning for recommendation of tasks in activities of daily living in smart homes. In: 2018 IEEE 42nd Annual Computer Software and Applications Conference (COMPSAC). pp. 317-322. IEEE (2018)

19. Reiss, A., Stricker, D.: Creating and benchmarking a new dataset for physical activity monitoring. In: Proceedings of the 5th International Conference on PErvasive Technologies Related to Assistive Environments. p. 40. ACM (2012)

20. Rialle, V., Duchene, F., Noury, N., Bajolle, L., Demongeot, J.: Health" smart" home: information technology for patients at home. Telemedicine Journal and EHealth 8(4), 395-409 (2002) 
21. Sahaf, Y.: Comparing sensor modalities for activity recognition. Ph.D. thesis, Washington State University (2011)

22. Salman, Y., Abu-Issa, A., Tumar, I., Hassouneh, Y.: A proactive multi-type context-aware recommender system in the environment of internet of things. In: Computer and Information Technology; Ubiquitous Computing and Communications; Dependable, Autonomic and Secure Computing; Pervasive Intelligence and Computing (CIT/IUCC/DASC/PICOM), 2015 IEEE International Conference on. pp. 351-355. IEEE (2015)

23. Sewak, M., Singh, S.: Iot and distributed machine learning powered optimal state recommender solution. In: Internet of Things and Applications (IOTA), International Conference on. pp. 101-106. IEEE (2016)

24. Yamada, N., Sakamoto, K., Kunito, G., Isoda, Y., Yamazaki, K., Tanaka, S.: Applying ontology and probabilistic model to human activity recognition from surrounding things. IPSJ Digital Courier 3, 506-517 (2007)

25. Yao, L., Sheng, Q.Z., Benatallah, B., Dustdar, S., Wang, X., Shemshadi, A., Kanhere, S.S.: Wits: an iot-endowed computational framework for activity recognition in personalized smart homes. Computing 100(4), 369-385 (2018)

26. Yao, L., Sheng, Q.Z., Dustdar, S.: Web-based management of the internet of things. IEEE Internet Computing 19(4), 60-67 (2015)

27. Yao, L., Sheng, Q.Z., Ngu, A.H.H., Li, X.: Things of interest recommendation by leveraging heterogeneous relations in the internet of things. ACM Trans. Internet Technol. 16(2), 9:1-9:25 (Mar 2016)

28. Yao, L., Sheng, Q.Z., Ruan, W., Gu, T., Li, X., Falkner, N., Yang, Z.: Rf-care: Device-free posture recognition for elderly people using a passive rfid tag array. In: Proceedings of the 12th EAI International Conference on Mobile and Ubiquitous Systems: Computing, Networking and Services on 12th EAI International Conference on Mobile and Ubiquitous Systems: Computing, Networking and Services. pp. 120-129. ACM (2015)

29. Zhou, S., Chu, C.H., Yu, Z., Kim, J.: A context-aware reminder system for elders based on fuzzy linguistic approach. Expert Systems with Applications 39(10), 9411 - 9419 (2012). https://doi.org/https://doi.org/10.1016/j.eswa.2012.02.124 